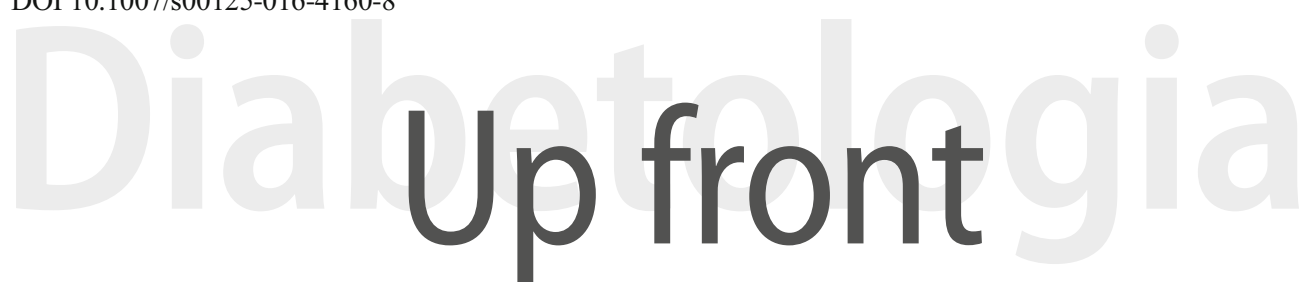

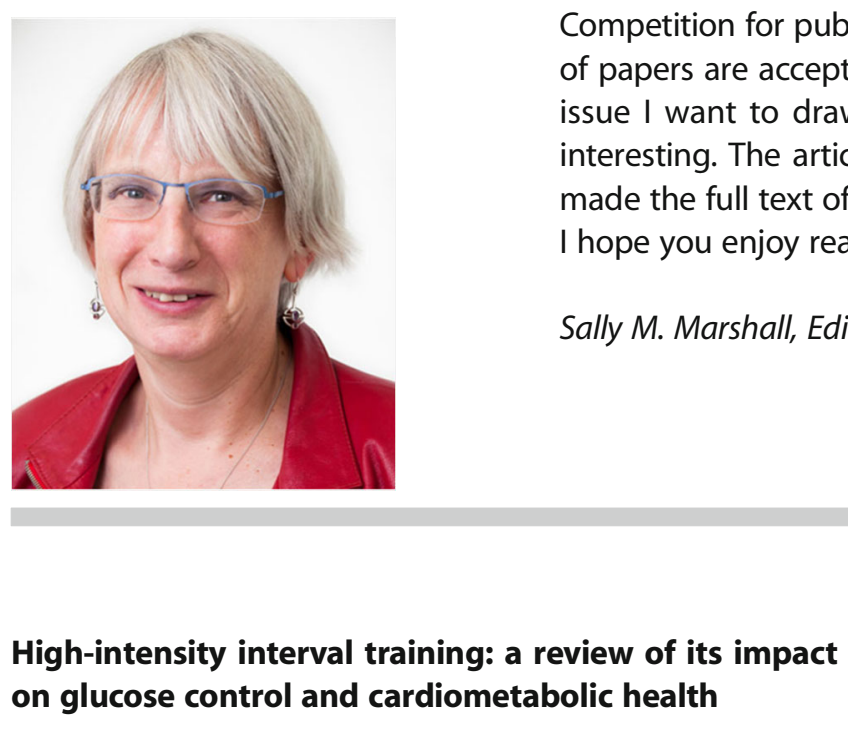

Sophie Cassidy, Christian Thoma, David Houghton, Michael I. Trenell

High-intensity intermittent training (HIIT) has gained much attention over the past decade because of its ability to induce a large physiological response with reduced time commitment, compared with other forms of exercise training. For this reason, the use of HIIT is increasing among clinical populations. In this issue, Cassidy et al aim to explore whether the acclaim surrounding HIIT is justified, by reviewing the current evidence for its impact on glucose control and cardiovascular health. They also report its safety, tolerability and the practical considerations required when adopting this form of exercise in a clinical setting. They conclude that, for the optimal clinical benefit (including improved glycaemic control and cardiovascular function) the value of HIIT appears likely to be adjunct to energy restriction.

\section{Serum endotrophin identifies optimal responders to PPAR $\gamma$ agonists in type 2 diabetes}

Morten A. Karsdal, Kim Henriksen, Federica Genovese, Diana J. Leeming, Mette J. Nielsen, Bente J. Riis, Claus Christiansen, Inger Byrjalsen, Detlef Schuppan

The extracellular matrix (ECM) is emerging to be more than just a passive scaffold: collagens are key structural components of the ECM, and during collagen degradation and processing, anti-angiogenic and anti-migratory cryptic fragments are released. The pro-peptide of type VI collagen is proteolytically spliced from the helical core during collagen maturation, with further cleavage of the pro-peptide resulting in endotrophin generation. Endotrophin has hormonal activities that are associated with insulin resistance and the metabolic syndrome. In this issue, Karsdal et al report that baseline endotrophin levels predicted the $\mathrm{HbA}_{1 \mathrm{c}^{-}}$and glucose-lowering response to two insulin sensitisers (peroxisome proliferator-activated receptor gamma (PPAR $\gamma$ ) agonists, balaglitazone and pioglitazone) in a phase III, type 2 diabetes study. Patients who had higher endotrophin levels, and thus responded better to these drugs, also had less severe side effects. These data indicate a need for a precision medicine approach to diabetes treatment and suggest that only a selected patient population may have a superior response with less adverse effects to PPAR $\gamma$ agonism. This article is the subject of a commentary in this issue by Sun et al.

\section{Exome-chip association analysis reveals an Asian- specific missense variant in PAX4 associated with type 2 diabetes in Chinese individuals}

Chloe Y. Y. Cheung, Clara S. Tang, Aimin Xu, Chi-Ho Lee, Ka-Wing Au, Lin Xu, Carol H. Y. Fong, Kelvin H. M. Kwok, Wing-Sun Chow, Yu-Cho Woo, Michele M. A. Yuen, JoJo S. H. Hai, Ya-Li Jin, Bernard M. Y. Cheung, Kathryn C. B. Tan, Stacey S. Cherny, Feng Zhu, Tong Zhu, G. Neil Thomas, Kar-Keung Cheng, Chao-Qiang Jiang, TaiHing Lam, Hung-Fat Tse, Pak-Chung Sham, Karen S. L. Lam

Conventional genome-wide association studies (GWAS) have identified a large number of common type 2 diabetes susceptibility variants that are predominately located at the 
intronic and intergenic regions. However, the functional consequences of such variants remain difficult to interpret. In this issue, Cheung, Tang et al conducted an exome-chip association analysis with a custom designed exome array, the Asian Exomechip. They report an association between type 2 diabetes and the Asian-specific missense variant (p.Arg192His) in PAX4. This variant may potentially decrease the transcription activity of $P A X 4$, impacting pancreatic beta cell differentiation, proliferation and insulin production. The risk allele of p.Arg192His was also associated with younger age at diabetes diagnosis. These findings suggest that PAX4 is a possible effector gene of the $7 q 32$ locus, which was previously identified by GWAS in Asians. They also provide supporting evidence for the role of $P A X 4$, a known gene for MODY, in the pathogenesis of common type 2 diabetes.

\section{Immunogenicity of human embryonic stem cell- derived beta cells}

Cornelis R. van der Torren, Arnaud Zaldumbide, Gaby Duinkerken, Simone H. Brand-Schaaf, Mark Peakman, Geert Stangé, Laura Martinson, Evert Kroon, Eugene P. Brandon, Daniel Pipeleers, Bart O. Roep

The treatment of type 1 diabetes by transplantation with a donor pancreas or pancreatic islets can result in durable remission of diabetes, but donors are rare. Glucose-responsive, insulin-secreting cells can be cultured from pancreatic endoderm derived from human embryonic stem cells (hESC), for an alternative and unlimited source of beta cells. However, their implantation into type 1 diabetic recipients may result in reactivation of the autoimmune process that caused destruction of the patient's own beta cells, while their donor origin may induce allograft rejection. In this issue, van der Torren et al report the immune reactions against hESC-derived pancreatic progenitor cells and endocrine cells, which were retrieved after in vivo differentiation in capsules in mice. The progenitor cells proved to be hypoimmunogenic, but immunogenicity changed during inflammation or their differentiation into endocrine cells, resulting in immune attack by alloantibodies, and alloand auto-reactive T cells. These data support the need for immune intervention or macro-encapsulation in the transplantation of hESC-derived pancreatic progenitor cells.

\section{Context-dependent memory following recurrent hypoglycaemia in non-diabetic rats is mediated via glu- cocorticoid signalling in the dorsal hippocampus}

Danielle M. Osborne, Kelsey E. O'Leary, Dennis P. Fitzgerald, Alvin J. George, Michael M. Vidal, Brian M. Anderson, Ewan C. McNay

Recurrent hypoglycaemia is a common side effect of insulin therapy and its potential impact on the brain is a major obstacle to patient compliance with optimal insulin therapy. A marked impact of recurrent hypoglycaemia on subsequent hippocampal memory processing has been previously shown in a rat model. In this issue, Osborne et al use the same model to identify specific molecular changes in the hippocampus after recurrent hypoglycaemia. They demonstrate that both altered memory processing and molecular changes are largely mediated by increased hippocampal glucocorticoid signalling during hypoglycaemia, which boosts subsequent performance when euglycaemic. Molecular changes included alterations in glutamate signalling ( $\alpha$-amino-3-hydroxy-5-methyl-4-isoxazolepropionic acid [AMPA] and $N$-methyl-D-aspartic acid [NMDA] receptors), serum/glucocorticoid-inducible kinase-1 (SGK1) and phosphorylated cyclic AMP response element binding (pCREB). The findings suggest that the impact of short-term recurrent hypoglycaemia on subsequent euglycaemic brain function may be beneficially adaptive. They also highlight a potential mechanism for alleviating diabetes-associated cognitive impairment.

All text supplied by the authors. 